Harrold, Liam (2021): „Der

Umgang mit der NS-Vergan-

genheit zwischen Kritik und

Tradtion. Historische Sinnbil-

dung in Leser*innenbriefen

zum Dokumentationsort der

Reichserntedankfeste am

Bückeberg." In: Demokratie

Dialog 9 (2021) S. 13-17.

doi:10.17875/gup2021-1769

\section{Der Umgang mit der NS-Vergangenheit zwischen Kritik und Tradition}

\author{
Historische Sinnbildung in \\ Leser*innenbriefen zum \\ Dokumentationsort der \\ Reichserntedankfeste am Bückeberg
}

Liam Harrold

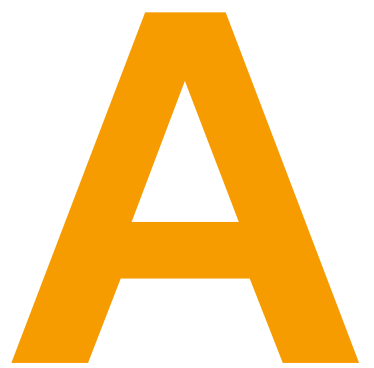

Is 2011 nach jahrelanger Debatte das Gelände des nationalsozialistischen Reichserntedankfestes am Bückeberg in der Nähe von Hameln vom Land Niedersachsen unter Denkmalschutz gestellt wurde, bestand schon die Idee, hier einen Dokumentations- und Lernort zu schaffen. Auf der Hangwiese am Bückeberg in der Gemeinde Emmertal im Landkreis Hameln-Pyrmont wurde zwischen 1933 und 1937 das Reichserntedankfest mitsamt militärischer Schauübungen für Propagandazwecke im Sinne der nationalsozialistischen "Volksgemeinschaft" unter der Gastgeberschaft des Reichspropagandaministers Joseph Goebbels veranstaltet. Die Diskussion um das Projekt Lernort, welches im Wesentlichen vom Hamelner Historiker Bernhard Gelderblom und dem Verein für regionale Kultur- und Zeitgeschichte Hameln vorangetrieben wurde, intensivierte sich ab 2017 und rief sowohl Befürworter*innen als auch Gegner*innen weit über die Landkreisgrenzen hinaus auf den Plan. Neben Diskussionsveranstaltungen etablierte sich als ein zentraler öffentlicher De- battenraum die Leser*innenbriefspalte der lokalen Deister-Weser-Zeitung (DeWeZet) sowie der Hannoverschen Allgemeinen Zeitung (HAZ).' Im Folgenden sollen Befunde einer im Rahmen einer Bachelorarbeit durchgeführten Untersuchung historischer Sinnbildung in Leser*innenbriefen vor dem Hintergrund folgender Fragen diskutiert werden: Wie stellt sich der Umgang mit der nationalsozialistischen Vergangenheit als geschichtskulturelles Phänomen in den Leser*innenbriefen dar und welche Orientierungsbedürfnisse kommen in ihnen zum Vorschein? Was bedeutet dies für gegenwärtige geschichtskulturelle Debatten zwischen Schlussstrich-Forderungen einerseits und sogenannter Aufarbeitung der NS-Vergangenheit andererseits? Um sich diesen Fragen zu nähern, muss jedoch zunächst die Funktion von Gedenk- und Dokumentationsorten skizziert werden.

1 Vgl. Sammlung Gelderblom: Presseberichte, Leser*innenbriefe und Zuschriften von 2002-2020, Hameln. Unveröffentlicht. 
Gedenk- und Dokumentationsorte und im weiteren Sinne Denkmäler können als geschichtskulturelles Phänomen begriffen werden. Geschichtskultur bezeichnet die gesellschaftliche bzw. kulturelle Auseinandersetzung mit sowie Formung bzw. - mit dem Historiker Jörn Rüsen gesprochen - Sinnbildung von Geschichte. ${ }^{2} \mathrm{Ge}-$ denk- und Dokumentationsorte erfüllen dementsprechend eine Orientierungsfunktion für die Gegenwart und Zukunft. Der Geschichtsdidaktiker Bodo von Borries stellt fest, dass Denkmäler „naturgemäß als ,Traditionen'“3 angelegt seien. Zudem weist von Borries auf die Problematik einer Dichotomie von Tradition und Kritik hin und stellt die Frage, inwiefern KZ-Gedenkstätten „kritisch" oder „traditional" sein können. "Kritisch” könne sowohl als Opposition zu den NS-Verbrechen als auch, auf die Gegenwart bezogen, in Bezug auf die fortdauernde Gefahr des Nationalismus und Faschismus verstanden werden. Doch auch mit „traditional“ könnte das Gedenken an die Opfer des Nationalsozialismus und die Pflicht zur Bekämpfung von Faschismus im Sinne des "Nie Wieder"4 von Theodor W. Adorno gemeint sein. Von Borries stellt fest: „Kritisch kann man sich nur gegen etwas wenden, was man als ,dominant', als ,Konvention', als ,Mainstream' definiert.." Diese Dichotomie kann so auch für Dokumentationsorte im Allgemeinen und den Bückeberg im Besonderen gelten. Dokumentationsorte erfüllen neben der appellativ mahnenden Funktion (Gedenkund Dokumentationsorte als Mahnmale) die zentrale Funktion historisch-politischer Bildung und werden daher oft auch Lernorte genannt. Dokumentationsorte verfolgen als außerschulische

2 Vgl. Rüsen, Jörn: Zeit und Sinn. Strategien historischen Denkens, Frankfurt a. M. 2012

3 Borries, Bodo von: Historisch Denken Lernen - Welterschließung statt Epochenüberblick. Geschichte als Unterrichtsfach und Bildungsaufgabe, Opladen/Farmington Hills 2008, S. 187.

4 Vgl. Adorno, Theodor W.: Erziehung nach Auschwitz (1966), in: Kadelbach, Gerd (Hrsg.): Erziehung zur Mündigkeit - Vorträge und Gespräche mit Hellmut Becker 1959 bis 1969, Frankfurt a. M. 1971

5 Borries, S. 187
Lernorte das Ziel, historisches Denken zu vermitteln. Das ist den Geschichtsdidaktikern Holger Thünemann und Johannes Jansen zufolge „ein gesellschaftlich bzw. geschichtskulturell gerahmter, sozial und kommunikativ vermittelter, aber zugleich individueller Prozess historischer Sinnbildung." ${ }^{16}$ Wie der Name schon nahelegt, erfüllen Dokumentationsorte auch die Funktion der Dokumentation von historischen Sachverhalten, die mit dem jeweiligen historischen Ort verbunden sind. NS-Gedenkstätten symbolisieren zudem eine besondere Verantwortung gegenüber den Opfern und Überlebenden der NS-Verbrechen und kommen einer ethisch-humanitären Funktion nach. In Anbetracht der geschichtskulturellen Entwicklung7 der Bundesrepublik in den letzten Jahrzehnten kann Gedenk- und Dokumentationsorten, die insbesondere die NS- und DDR-Geschichte thematisieren, ein Bedeutungszuwachs attestiert werden. Dies zeigt sich beispielsweise in der Fülle an Initiativen zur Schaffung neuer Gedenk- und Dokumentationsorte, für die auch die Diskussion um den Bückeberg bei Hameln ein Exempel darstellt.

Die Debatte um den Bückeberg bzw. den historischen Ort der Reichserntedankfeste begann Ende der 1990er Jahre, nachdem die Gemeinde Emmerthal schon in den 1980er Jahren eine Wohnbebauung am Rande des Geländes zuließ, die in den 1990ern auf dem ehemaligen Festplatz noch einmal erweitert wurde. Zudem wurde 1991 eine Bepflanzung der Überreste der oberen Rednertribüne und des östlichen Hangs vorgenommen mit der Begründung, man wolle verhindern, dass an diesem Ort zu Hitlers Geburtstag von Rechts-

6 Vgl. Thünemann, Holger/Jansen, Johannes: Historisches Denken lernen, in: Bracke, Sebastian et al:: Theorie des Geschichtsunterrichts, Schwalbach/ Ts. 2018 , S. 94.

7 Zur historischen Entwicklung sowie zur Theorie und Praxis von Gedenkstätten vgl. auch: Gryglewski, Elke et al. (Hrsg.): Gedenkstättenpädagogik. Kontext, Theorie und Praxis der Bildungsarbeit zu NS-Verbrechen, Berlin 2015 
radikalen Blumen niedergelegt würden. ${ }^{8}$ Die von Bernhard Gelderblom erarbeitete Ausstellung „Ein Volk dankt seinem (Ver)führer. Die Reichserntedankfeste auf dem Bückeberg 1933-1937" wurde 1999 erstmals gezeigt und fand auch überregional Beachtung. So wurde sie 2001 im Braunschweigischen Landesmuseum ausgestellt und von Abendvorträgen und einer Publikation gerahmt. ${ }^{9}$ Auch die niedersächsische Landeszentrale für politische Bildung veranstaltete 2002 eine Tagung zur überregionalen Bedeutung des Geländes, auf der sich die Teilnehmer*innen gegen eine Rekonstruktion des Festplatzes, aber ausdrücklich dafür aussprachen, den Ort unter Denkmalschutz zu stellen. ${ }^{10}$ So wurde 2002 auf Initiative Gelderbloms der Denkmalschutz beantragt, dem sich eine bis 2011 andauernde Debatte anschloss, die sich mit der Denkmalschutzfrage für den historischen Ort der Reichserntedankfeste befasste und im Zuge derer sich in der regionalen Presse auch kritische Stimmen zu Wort meldeten. So befürchteten Gegner*innen unter anderem die mögliche Entstehung „eine[r] ,Kultstätte' für politisch rechte Gruppierungen"11. 2011 wurde das Gelände dann als Kulturdenkmal unter Denkmalschutz gestellt, nachdem 2009 ein Expertensymposium aus Historiker*innen, Denkmalpfleger*innen sowie Landschaftsarchitekt*innen dem Ort eine nationale Bedeutung als Kulturdenkmal im Kontext der Aufarbeitung der NS-Geschichte zugesprochen hatte. ${ }^{12}$ Der Entwurf

8 Vgl. Gelderblom, Bernhard: Die NS-Reichserntedankfeste auf dem Bückeberg 1933-1937. Aufmarsch der Volksgemeinschaft und Massenpropaganda, Holzminden 2018, S. $181 \mathrm{f}$

9 Vgl. Biegel, Gerd/Otte, Wulf: Vorträge zur Ausstellung. Ein Volk dankt seinem (Ver)führer. Die Reichserntedankfeste auf dem Bückeberg 1933-1937, Braunschweig 2002.

10 Vgl. Gelderblom, S. 183

11 Hummel, Juliane/Keller, Rolf: Der Bückeberg bei Hameln. Ein langer Weg zum Kulturdenkmal und Informations- und Lernort, in: Gedenkstättenrundbrief 174 (2014), S. 26-31., hier S. 27.

12 Vgl. ebd., S.27 f für einen Dokumentations- und Lernort am Bückeberg wurde am 24. November 2017 bei einer Bürger*innenveranstaltung in der Gemeinde vorgestellt. Seitdem wurden Gelderblom zufolge ca. 200 Leser*innenbriefe in der Deister- und Weserzeitung (DeWeZet), der regionalen Tageszeitung, publiziert. $^{13}$ Eigene Sichtungen dieser Sammlung belegen zudem diverse Leser*innenbriefe auch in der Hannoverschen Allgemeinen Zeitung (HAZ) als bedeutender überregionaler Tageszeitung. Diese Briefe hätten es laut Gelderblom geschafft, "die öffentliche Diskussion in einem hohen Maße zu bestimmen und viele Menschen in Emmerthal und im Landkreis Hameln-Pyrmont glauben zu machen, es handele sich um ein ,maßloses' und überaus kostspieliges Projekt." ${ }^{14}$

Für die diesem Artikel zugrundeliegende Untersuchung wurden 16 Leser*innenbriefen (die aus einer Sammlung von über 147 hauptsächlich in den Jahren 2017 bis 2019 publizierten Leser*innenbriefen ausgewählt wurden) auf ihren historischen Sinnbildungsgehalt mithilfe der Theorie historischen Erzählens von Jörn Rüsen ${ }^{15}$ untersucht; einige Auszüge daraus sollen nun hier präsentiert werden. Rüsen charakterisiert die „Erinnerungsleistung des Geschichtsbewusstseins formal als Erzählen einer Geschichte"16 und begreift das Geschichtsbewusstsein als den Ort, an dem Sinnbildung über Zeiterfahrung vollzogen wird. ${ }^{17}$ Die jeweilige historische Sinnbildung in den Leser*innenbriefen zeigt also Blitzlichter des Geschichtsbewusstseins der Autor*innen auf. Die Briefe sind folglich ein Akt der Kommunikation des Geschichtsbewusstseins und werden so Teil von Geschichtskultur, die hier mit Rüsen als „praktisch wirksame Artikulation von Geschichts-

13 Vgl. Gelderblom, S. 189

14 Ebd., S. 185.

15 Vgl. bspw. Rüsen, Jörn: Historisches Erzählen, in: Bergmann, Klaus et al. (Hrsg): Handbuch der Geschichtsdidaktik, Seelze-Velber 1997, S.57-63 und ders.: Historik. Theorie der Geschichtswissenschaft, Köln u. a. 2013.

16 Rüsen: Zeit und Sinn, S. 241.

17 Vgl. ebd., S. 16 
bewusstsein im Leben einer Gesellschaft" ${ }^{\text {"18 }}$ verstanden werden kann. Die Leser*innenbriefe und deren Argumentationen sind mit ihrer Veröffentlichung Teil einer größeren geschichtskulturellen Debatte geworden und haben sogar einen wesentlichen Beitrag zur öffentlichen Meinungsbildung geleistet. ${ }^{19}$

Zudem kamen in der Debatte um den Dokumentationsort zwei zentrale Orientierungsbedürfnisse bei den Briefautor*innen zum Ausdruck: Erstens gibt es diejenigen, die sich positiv zum Dokumentations- und Lernort positionieren und an eine Gedenk- und Erinnerungstradition nach 1945 anknüpfen, diese als moralisch notwendig und als Teil demokratischer Traditionen verstehen. Zweitens gibt es diejenigen, die mit dem Erinnern an den Nationalsozialismus abschließen wollen, einen Schlussstrich ziehen möchten und die zwölf Jahre NS-Herrschaft als eine zu vernachlässigende Episode deutscher Geschichte verstehen. Während beide in die Zukunft schauen, tun sie dies jedoch auf fundamental unterschiedliche Weise. Der kategorische Imperativ Adornos, "dass Auschwitz nie wieder sei", wird in einer demokratischen Tradition als Bildungsauftrag des Dokumentations- und Lernortes verstanden und zum Postulat der Befürworter. Während die Schlussstrich-Fraktion sich in ihren Leser*innenbriefen vermeintlich "gegenwärtigeren Problemen" wie Kita, Schule, schlechte Haushaltslage öffentlicher Einrichtungen und des Staates zuwendet, bedient sie sich einer NS-relativierenden Sprache und Argumenten, die in ihren schärfsten Ausprägungen an rechte Narrative erinnern. So heißt es in einem Leserbrief beispielsweise: "Ich werde den Verdacht nicht los, dass uns Deutschen ein ewiges Schuldgefühl auferlegt werden soll, um die millionenfache Migration von größtenteils muslimischen Wirtschaftsmigranten zu rechtfertigen." ${ }^{20}$ Die angeführten Argumentationen ge-

18 Ebd., S. 235.

19 Vgl. Gelderblom, S. 185.

20 Vgl. Fiegert, Rainer: Investition in marode Schulen nicht sinnvoller?, in: Deister-Weser-Zeitung, 17.01.2018, S. 14. gen die Errichtung des Dokumentationsortes sind indes nicht neu und müssen im Kontext ähnlicher Debatten wie zum Beispiel der um die Errichtung einer Gedenkstätte im ehemaligen KZ Dachau gesehen werden. ${ }^{21}$

Historische Sinnbildung als solche manifestiert sich in den Leser*innenbriefen im bewährten Muster des Für und Widers: Die Einen argumentieren für den Dokumentationsort und sehen das Postulat des "Nie Wieder" Adornos und die Entwicklung der Erinnerungskultur von einem gegenkulturellen Projekt hin zu einer überwiegend gesellschaftlich akzeptierten und staatlichen (oder staatlich geförderten) Erinnerungskultur auf ihrer Seite..2 Die Anderen argumentieren gegen den Dokumentationsort und kritisieren wahlweise das Verfahren bis zur Entscheidungsfindung, mangelnde Bürger*innenbeteiligung, die Kosten oder die Erinnerungskultur in Gänze. Einschränkend muss festgehalten werden, dass es nicht zwingend bei den beiden skizzierten Orientierungsbedürfnissen bzw. Sinnbildungen bleibt. Denn: Nicht alle Briefe positionieren sich eindeutig, manche suchen gar einen Mittelweg oder sind mit „Kompromiss" überschrieben. Diese vermittelnden Positionen waren jedoch stark in der Unterzahl. Zusammengenommen zeigen die Briefe, dass geschichtskulturelle Debatten und geschichtspolitische Kämpfe um Deutungshohei-

Auch in: Sammlung Gelderblom.

21 Zu Dachau als Beispiel einer anderen geschichtskulturellen Debatte, die der Schaffung einer Gedenkstätte vorausging und diese begleitet hat, vgl. Siebeck, Cornelia: 50 Jahre „arbeitende“ NS-Gedenkstätten in der Bundesrepublik. Vom gegenkulturellen Projekt zur staatlichen Gedenkstättenkonzeption - und wie weiter?, in: Gryglewski, Elke et al. (Hrsg.): Gedenkstättenpädagogik. Kontext, Theorie und Praxis der Bildungsarbeit zu NS-Verbrechen, Berlin 2015., S. 19-43, hier S. 19 f.

22 Siebeck, Cornelia: 50 Jahre „arbeitende” NS-Gedenkstätten in der Bundesrepublik. Vom gegenkulturellen Projekt zur staatlichen Gedenkstättenkonzeption - und wie weiter? in: Gryglewski, Elke et al. (Hg.): Gedenkstättenpädagogik. Kontext, Theorie und Praxis der Bildungsarbeit zu NS-Verbrechen, Berlin 2015, S. 19-43, hier S. $33 \mathrm{ff}$. 
ten immer wieder neu ausgetragen werden müssen. Die hier untersuchte Debatte und damit auch die Leser*innenbriefe reihen sich in eine Entwicklung der Erinnerungskultur ein, die von der die Auschwitz-Prozesse der 1960er Jahre begleitenden öffentlichen Debatte über die Ausstrahlung der US-amerikanischen Serie "Holocaust" im westdeutschen Fernsehen, über die Rede des damaligen Bundespräsidenten Richard von Weizsäcker 1985 bis hin zu jüngeren Debatten wie der um das Holocaust-Mahnmal in Berlin, das der Politiker Björn Höcke (AfD) 2017 als „Mahnmal der Schande" bezeichnete, reicht.

Erinnerungskulturelle Debatten sind oft durch auseinanderklaffende Meinungen zum gegenwärtigen Umgang mit der Vergangenheit geprägt. Zwei zentrale Begriffe, die in (geschichts-)politischen und geschichtskulturellen Debatten immer wieder neu verhandelt werden (müssen), sind, wie bereits oben erwähnt, Tradition und Kritik. Wer übt woran wie Kritik und warum? Welches Traditionsverständnis haben verschiedene Generationen, Bevölkerungsgruppen etc.? Denn Traditionen bzw. traditionale Erzählungen können sich unterscheiden und verändern, je nachdem, welche historischen Bezugspunkte man wählt. Historische Sinnbildung bleibt zudem weiterhin eine der zentralen geschichtstheoretischen Kategorien, weswegen die Frage, wie und warum Menschen Geschichte schreiben bzw. erzählen, immer wieder neu gestellt und etablierte Antworten überprüft werden müssen. Denn so wie Gesellschaft ist auch Geschichtskultur stetiger Veränderung im Zeitverlauf zwischen Vergangenheit, Gegenwart und Zukunft unterworfen. Historische Sinnbildung und unsere gegenwärtigen Orientierungsbedürfnisse prägen dabei unser Verhältnis zur Vergangenheit. Sie beeinflussen, wie wir Geschichte schreiben, und prägen damit nicht nur unser Geschichtsverständnis, sondern auch gegenwärtige und zukünftige gesellschaftliche und (geschichts-) politische Debatten, wie sich an der kontroversen Diskussion um den Lern- und Dokumentationsort am Bückeberg eindrucksvoll belegen lässt.
Literatur:

Adorno, Theodor W.: Erziehung nach Auschwitz. (1966), in: Kadelbach, Gerd (Hrsg): Erziehung zur Mündigkeit - Vorträge und Gespräche mit Hellmut Becker 1959 bis 1969, Frankfurt a. M. 1971.

Biegel, Gerd/Otte, Wulf: Vorträge zur Ausstellung. Ein Volk dankt seinem (Ver)führer. Die Reichserntedankfeste auf dem Bückeberg 1933-1937, Braunschweig 2002.

Borries, Bodo von: Historisch Denken Lernen - WelterschlieBung statt Epochenüberblick. Geschichte als Unterrichtsfach und Bildungsaufgabe, Opladen/Farmington Hills 2008.

Bracke, Sebastian/Flaving, Colin/Jansen, Johannes/Köster, Manuel/Lahmer-Gebauer, Jennifer/Lankes, Simone/Spieß, Christian/Thünemann, Holger/Wilfert, Christoph/Zülsdorf-Kersting, Meik (Hrsg.): Theorie des Geschichtsunterrichts, Schwalbach/Ts. 2018.

Gelderblom, Bernhard: Die NS-Reichserntedankfeste auf dem Bückeberg 1933-1937. Aufmarsch der Volksgemeinschaft und Massenpropaganda, Holzminden 2018.

Gryglewski, Elke/Haug, Verena/Kößler, Gottfried/ Lutz,

Thomas/Schikorra, Christa (Hrsg.): Gedenkstättenpädagogik. Kontext, Theorie und Praxis der Bildungsarbeit zu NS-Verbrechen, Berlin 2015.

Hummel, Juliane/Keller, Rolf: Der Bückeberg bei Hameln. Ein langer Weg zum Kulturdenkmal und Informations- und Lernort, in: Gedenkstättenrundbrief 174 (2014), S. 26-31.

Rüsen, Jörn: Historisches Erzählen, in: Bergmann, Klaus/Fröhlich, Klaus/ Kuhn, Annette (Hrsg): Handbuch der Geschichtsdidaktik, Seelze-Velber 1997, S. 57-63.

Rüsen, Jörn: Zeit und Sinn. Strategien historischen Denkens, Frankfurt a. M. 2012

Rüsen, Jörn: Historik. Theorie der Geschichtswissenschaft, Köln u. a. 2013.

Siebeck, Cornelia: 50 Jahre „arbeitende” NS-Gedenkstätten in der Bundesrepublik. Vom gegenkulturellen Projekt zur staatlichen Gedenkstättenkonzeption - und wie weiter? in: Gryglewski, Elke et al. (Hg.): Gedenkstättenpädagogik. Kontext, Theorie und Praxis der Bildungsarbeit zu NS-Verbrechen, Berlin 2015, S. 19-43.

\section{Quellen:}

Sammlung Gelderblom: Presseberichte, Leser*innenbriefe und Zuschriften von 2002-2020, Hameln. Unveröffentlicht

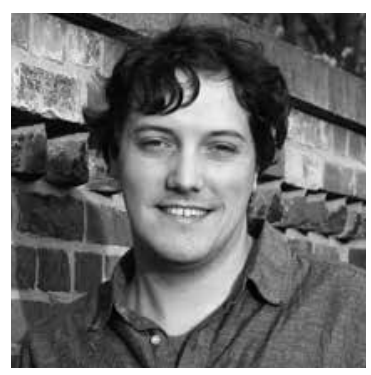

Liam Harrold, geb. 1995, Studium der Geschichte und Philosophie an der Leibniz Universität Hannover, seit April 2021 im Master Geschichte ebenfalls am Historischen Seminar in Hannover. Seine Forschungsschwerpunkte sind deutsche Umweltgeschichte, Geschichte des Nationalsozialismus und Holocaustforschung, deutsche und europäische Erinnerungs- und Geschichtskultur sowie Geschichtstheorie. 